\title{
Evolução histórica da medicina veterinária preventiva e saúde pública
}

\author{
Historical evolution of preventive veterinary medicine and public health
}

\author{
Márcia Regina Pfuetzenreiter ${ }^{1}$ Arden Zylbersztajn ${ }^{2}$ \\ Fernando Dias de Avila-Pires ${ }^{3}$
}

\section{RESUMO}

\begin{abstract}
O artigo aborda a evolução histórica da participação da medicina veterinária na saúde pública e as ações desempenhadas pelo médico veterinário nesse campo de atuação. As etapas do desenvolvimento do pensamento preventivo em Medicina Veterinária são descritas desde o surgimento das atividades relacionadas à medicina veterinária preventiva, até o início do trabalho do médico veterinário voltado para a população. O desenvolvimento do conceito de saúde pública veterinária foi acompanhado desde a criação do termo até a formulação do conceito atual. As funções do médico veterinário na saúde pública e a contribuições da Medicina Veterinária para a saúde humana são destacadas com a apresentação do cenário atual e das tendências para a Medicina Veterinária Preventiva e Saúde Pública. Alguns aspectos da educação veterinária relacionados com a saúde pública são discutidos tendo em vista questões de maior relevância social na atualidade e para o futuro.
\end{abstract}

Palavras-chave: medicina veterinária, saúde pública, medicina preventiva, evolução histórica.

\section{ABSTRACT}

The historical evolution of the insertion of veterinary medicine in public health and the actions performed by doctors in veterinary medicine in this area are presented. The stages of development of the preventive perspective in Veterinary Medicine are described from its origins, as activities related to preventive veterinary medicine, to the beginning of the work of doctors in veterinary medicine directed to human population. The development of the concept of public health in veterinary was followed since its creation to present days. The functions of doctors in veterinary medicine are stressed with the presentation of the nowadays situation and tendencies for Preventive Veterinary Medicine and Public Health. Some aspects of veterinary education related with public health are discussed considering the relevant questions it poses both for present days and the future.

Key words: veterinary medicine, public health, preventive medicine, historical evolution.

\section{INTRODUÇÃO}

A pesquisa histórica aqui apresentada, feita por meio de um trabalho bibliográfico documental traça a evolução histórica da Medicina Veterinária Preventiva e Saúde Pública desde os seus primórdios. O surgimento do conceito de saúde pública veterinária foi analisado, bem como as atividades realizadas pelo médico veterinário dentro desse âmbito, até chegar ao cenário atual, apontando as tendências e desafios para o setor.

A prática veterinária tem sido muito voltada aos aspectos populacionais e preventivos e muitas táticas utilizadas para o combate de enfermidades em populações humanas foram contribuições prestadas pela Medicina Veterinária.

Há dois tipos de prática da Medicina Veterinária que estão direcionadas para a medicina populacional. Uma delas é a Medicina Veterinária Preventiva que está ligada à saúde humana por aplicar conhecimentos da epidemiologia para prevenir as enfermidades animais e melhorar a produção de alimentos. O segundo tipo de prática veterinária voltada para a medicina populacional é a saúde pública, que foi primeiramente desenvolvida por meio da higiene de alimentos.

\section{O SURGIMENTO DAS ATIVIDADES DE MEDICINA VETERINÁRIA PREVENTIVA}

SCHWABE (1984) descreve as ações praticadas pela Medicina Veterinária Preventiva dividindo-as em cinco fases e as considera

${ }^{1}$ Graduação em Medicina Veterinária e Doutorado em Educação, Departamento de Medicina Veterinária Preventiva e Tecnologia da Universidade do Estado de Santa Catarina, Av. Luiz de Camões, 2090, 88530-000, Lages, SC, Email: marcia@ cav.udesc.br.

${ }^{2}$ Licenciatura em Física e Doutorado em Ensino de Ciências, Departamento de Física, Universidade Federal de Santa Catarina (UFSC), Campus Universitário, 88040-900, Florianópolis, SC, Email: arden@fsc.ufsc.br

${ }^{3}$ Bacharelado e Licenciatura em História Natural e Doutorado em Ciências Biológicas, Departamento de Saúde Pública, UFSC, Campus Universitário, 88040-900, Florianópolis, SC. 
aparentadas com as atividades relacionadas à "doença animal":

\section{Fase de ações locais}

Este período tem seu princípio na préhistória e continua até o primeiro século da era cristã. Os primeiros esforços dirigidos contra a doença animal que se tem conhecimento foram descritos nas antigas civilizações da Suméria, Egito e Grécia, com referências a curandeiros de animais antes da era cristã. Esse tipo de ocupação acompanhou o surgimento da civilização urbana, desenvolvimento que dependeu da habilidade das populações rurais em produzir alimentos em quantidade suficiente para sua subsistência, fazendo uso da força animal. Ao lado do tratamento médico, cirúrgico e obstétrico individual, duas outras táticas eram aplicadas localmente para o controle das enfermidades animais, antes ainda que tivesse sido desenvolvida a teoria do contágio: o emprego da quarentena (segregação dos animais doentes dos sadios) e o sacrifício de animais enfermos.

\section{Fase militar}

Essa fase tem seu início no primeiro século da era cristã. A expansão das nações levou aos esforços no controle de doenças animais em larga escala. Houve a criação de estruturas organizadas de pessoas que curavam os animais dentro dos exércitos, pela importância militar que o cavalo assumia. Durante esse longo período de serviços veterinários que abrangeu a Idade Média e o Renascimento, os avanços no controle de doenças se limitaram ao aperfeiçoamento das técnicas básicas do diagnóstico clínico com o desenvolvimento da habilidade de diferenciar as combinações dos sinais de doenças específicas. Essa quarta tática para o controle das enfermidades dos animais estava associada à melhoria na organização de infra-estrutura dos serviços.

\section{Fase da polícia sanitária animal}

A terceira fase começa em 1762 - com a criação da primeira escola de veterinária. O início dessa fase se precipitou pelos problemas econômicos ocasionados pelo irrompimento de enfermidades atingindo um grande número de animais na Europa. Essa crise foi germinal para o estabelecimento da primeira escola de medicina veterinária separada da medicina humana. Os líderes militares reconheceram o potencial de tais esforços educacionais organizados e muitos estudantes das primeiras escolas eram oficiais militares. Nessa fase, houve o estabelecimento de centros organizados de tratamento veterinário, primeiramente como parte das escolas de veterinária e, mais tarde, como serviços separados.

Duas novas táticas para o controle de enfermidades animais foram adotadas: a higiene (quinta tática) e o controle sobre o abate de animais (sexta tática). O controle sanitário incluía os locais de produção de animais e os matadouros, com o objetivo de combater as doenças animais e também as enfermidades humanas que estavam sendo associadas a alimentos de origem animal. Essas ações forneceram diretamente a base para os primeiros esforços direcionados à saúde pública. A aplicação dessas táticas representou uma oportunidade para o trabalho educacional dos proprietários de animais. Observou-se que uma das principais falhas dos programas veterinários para o controle de enfermidades não estaria nas deficiências técnicas dos programas, mas nas deficiências da comunicação com o público.

Fase das campanhas ou ações coletivas

Os anos 80 do século XIX inauguram essa fase, com as observações e experimentos sobre o anthrax por Delafond - diretor da Escola de Veterinária de Alfort (segunda escola de veterinária fundada no mundo) - e pelos conhecidos trabalhos de Pasteur, Chauveau, Koch e Salmon. Esses nomes e outros conduziram à "revolução microbiológica" como resultado da compreensão das formas de contágio, que forneceu a base para uma nova abordagem para a investigação de doenças na busca e identificação de seus agentes etiológicos. Foram iniciados programas de ações governamentais no combate às infecções dos animais de fazenda. Durante essa fase houve grande sucesso no controle de doenças, o que abriu a possibilidade para a criação de animais em produção intensiva.

Nessa fase, foi introduzida uma outra tática para a prevenção e controle de enfermidades que consiste em ações populacionais como o diagnóstico, a imunização e a terapia em escala populacional, além de alguns procedimentos em ecologia aplicada como o controle de vetores. Muitas dessas medidas, primeiramente visualizadas e praticadas pelos veterinários, foram posteriormente extrapoladas e se mostraram bem sucedidas para problemas similares em saúde pública. A aplicação dessas medidas permitiu o uso rápido e sistemático de outros procedimentos como a quarentena, sacrifício de animais reagentes e desinfecção local.

O controle de vetores surgiu como uma medida preventiva única, sem precedentes como resultado dos estudos epidemiológicos de Salmon 
juntamente com Kilborne, Smith e Curtice. Esses pesquisadores foram os primeiros a demonstrar a transmissão de um microrganismo por meio de artrópodes sobre a febre do gado no Texas piroplasmose bovina ou babesiose. Ao lado das táticas citadas para essa fase, que durou até os anos de 1960, está a educação em saúde dos proprietários dos animais.

Fase de vigilância e ações coletivas

O surgimento da teoria sobre os agentes etiológicos de doença pela revolução microbiológica marcou uma fase muito produtiva para a Medicina Veterinária Preventiva. No entanto, observou-se que outros fatores intervinham no aparecimento das enfermidades, sendo necessária uma abordagem mais ampla do problema. Muitas vezes, a presença do agente etiológico é necessária, mas não suficiente para explicar o aparecimento das enfermidades. Essa constatação gerou uma crise na Medicina Veterinária Preventiva que se instalou no início dos anos de 1950 pela verificação de vários aspectos (SCHWABE, 1984): a) apesar de serem efetuadas campanhas contra uma série de enfermidades, houve uma redução das mesmas, mas sem produzir sua eliminação; b) o custo para o controle de muitas enfermidades era muito grande; c) ausência de conhecimentos para o controle de algumas doenças; d) incapacidade em lidar com novas situações práticas que surgiam na criação intensiva.

Em resposta a essa crise, surgiu a "revolução epidemiológica", com a compreensão de que cada situação requer análise dos fatores que interagem para a ocorrência de doenças. A epidemiologia, que focaliza seus estudos sobre populações, foi introduzida na Medicina Veterinária Preventiva por meio da Saúde Pública para auxiliar sua prática. O diagnóstico epidemiológico passou a constituir uma nova tática para o controle de enfermidades. Essa fase teve seu início na década de 1960 e continua até os dias de hoje (SCHWABE, 1984).

Neste período, a epidemiologia começou a ser reconhecida como campo de estudo e muitos médicos e médicos veterinários se tornaram conscientes da aptidão destes últimos para trabalhar em saúde pública. O ingresso simultâneo dos profissionais da Medicina Veterinária no campo das doenças transmissíveis e nos serviços médicos preventivos foi permitido pelo reconhecimento dos seus conhecimentos e habilidades em medicina populacional e também pela importância das zoonoses, que perfazem $80 \%$ das doenças transmissíveis em humanos. Essas habilidades dos veterinários e esses atributos que eles podem levar para a saúde pública fazem desta profissão um elo de ligação entre o setor da agricultura e da saúde humana (ORGANIZACIÓN PANAMERICANA DE LA SALUD, 1975).

\section{A SAÚdE PÚBLICA VETERINÁRIA}

A saúde pública é a segunda área da prática veterinária voltada para as populações. ROSEN (1994) descreve algumas ações ligadas a essa área na idade média. Nessa época, não havia planejamento urbano e as moradias se apinhavam sob os muros de proteção das cidades onde os habitantes conservavam hábitos da vida no campo. Animais como porcos, gansos e patos eram criados nas casas, o que causava incômodo pelo acúmulo de excrementos. Para resolver o problema, os municípios criaram uma série de regulamentos, que incluía a construção de instalações para os animais e a criação de matadouros municipais. As medidas para o controle de alimentos já eram tomadas na época, porém em certas cidades a carne de animais doentes era enviada para hospitais.

Após a fundação das primeiras escolas de Medicina Veterinária, na segunda metade do século XVIII seguiram-se dois movimentos. O primeiro deles estava destinado a deter as epidemias que atingiam o gado naquela época e o segundo voltado para reduzir os riscos para a saúde humana ao abate indiscriminado de animais para comercialização (SCHWABE, 1984).

O início das atividades da Medicina Veterinária em Saúde Pública ocorreu no século XIX, na indústria da carne. Robert von Ostertag na Alemanha e Daniel E. Salmon nos Estados Unidos da América deram início ao que se conhece atualmente como proteção dos alimentos (ORGANIZACIÓN PANAMERICA DE LA SALUD, 1975).

A importância da Medicina Veterinária para a saúde humana coincidiu com o crescente reconhecimento entre os núcleos de estudiosos de médicos e veterinários europeus que desenvolviam pesquisas médicas comparadas em parceria nas áreas de anatomia e fisiologia. Esses estudos ocorreram particularmente nas escolas de veterinária francesas na primeira metade do século XIX e o prosseguimento dessas pesquisas forneceu os princípios para a elaboração da "revolução microbiológica". O incremento da pesquisa médica comparada no final do século XIX propiciou uma forte ligação entre a Medicina Veterinária e a Medicina Humana e 
influenciou o desenvolvimento de uma tradição educacional em algumas escolas de veterinária mais intimamente ligada aos interesses da medicina humana que da agricultura (SCHWABE, 1984).

SCHWABE (1984) descreve os períodos de atividade da saúde pública dentro da Medicina Veterinária. $\mathrm{O}$ primeiro período teve como alicerce a higiene de alimentos e foi a partir dessa base que alguns poucos veterinários assumiram posições administrativas nos programas de saúde pública de vários países, no final do século XIX e início do século XX. Seguiu-se um intervalo de relativa estabilidade da participação veterinária no trabalho de saúde pública que durou até a Segunda Guerra Mundial.

A segunda fase da Medicina Veterinária na saúde pública, que teve seu início após a Segunda Guerra, se caracterizou pelo trabalho voltado para a população com o uso da epidemiologia no desenvolvimento de programas de controle de zoonoses pelas agências de saúde pública. Como conseqüência da interação com profissionais da medicina humana, os médicos veterinários começaram a ocupar várias posições nas áreas técnicas e administrativas da saúde pública.

Em 1944, a Organização Panamericana de Saúde começou a contratar veterinários como consultores. Em 1946, a conferência de estruturação da Organização Mundial de Saúde recomendou a criação de uma seção de saúde veterinária, que foi estabelecida em 1949 (VIANNA PAIM \& CAVALCANTE DE QUEIROZ, 1970). A evolução da saúde pública veterinária será vista a seguir, sendo utilizados como base os documentos produzidos na época para a pesquisa histórica empreendida.

O termo saúde pública veterinária foi utilizado oficialmente pela primeira vez em 1946, durante um encontro que incumbia a OMS de fornecer uma estrutura conceitual e programática para aquelas atividades de saúde pública que envolvem a aplicação do conhecimento em Medicina Veterinária direcionado para a proteção e promoção da saúde humana. Na primeira reunião da OMS/FAO o termo foi assim definido: "A saúde pública veterinária compreende todos os esforços da comunidade que influenciam e são influenciados pela arte e ciência médica veterinária, aplicados à prevenção da doença, proteção da vida, e promoção do bem-estar e eficiência do ser humano". (WORLD HEALTH ORGANIZATION, 1951).

O documento aponta que o termo saúde pública veterinária era relativamente novo na língua inglesa-veterinary public health, mas já havia ganho grande aceitação. Em nota de rodapé, o grupo recomenda as traduções para alguns idiomas: para o francês - hygiène publique vétérinaire, para o espanhol - salud publica veterinária, em italiano sanità pubblica veterinária, e, finalmente em português - saúde pública veterinária.

O médico veterinário se incorpora muito facilmente ao grupo de profissionais de saúde por estar habituado a proteger a população contra as enfermidades coletivas. O tipo de formação recebida pelo veterinário está em harmonia com o conceito de saúde pública, que considera todos os fatores que determinam a saúde coletiva, sem limitar-se às necessidades do indivíduo. $\mathrm{O}$ informe complementa que em muitas escolas de veterinária, a medicina preventiva - que se ocupa em combater as enfermidades animais - forma uma parte tão importante do programa quanto a patologia, a clínica e a cirurgia veterinária (ORGANIZACIÓN MUNDIAL DE LA SALUD, 1957).

As atividades da saúde pública veterinária citadas são: as zoonoses, a higiene dos alimentos e os trabalhos de laboratório, de biologia e as atividades experimentais. O informe assinala que a luta contra as zoonoses se constitui em uma das principais atividades da saúde pública veterinária. Essas enfermidades constituem um importante fator de morbidade e pobreza, pelas infecções agudas e crônicas que causam aos seres humanos e pelas perdas econômicas ocasionadas na produção animal. A prevenção e a eliminação desse tipo de enfermidade no homem dependem, em grande parte, das medidas adotadas contra essas doenças nos animais. No texto, argumenta-se que as ações de combate não podem ser adotadas independemente pelas autoridades sanitárias e agrícolas e a melhor maneira para enfrentar o problema seria coordenar os esforços dos serviços de saúde e de agricultura por meio da saúde pública veterinária (ORGANIZACIÓN MUNDIAL DE LA SALUD, 1957).

No Segundo Comunicado Técnico de Especialistas em Zoonoses (WORLD HEALTH ORGANIZATION, 1959) foi reconhecida a existência de mais de cem zoonoses, o que fez com que aumentasse a importância dos programas de prevenção, controle e erradicação dessas enfermidades.

Dando seqüência à série de reuniões efetuadas para discutir temas ligados à saúde pública veterinária, especialistas do comitê da FAO/OMS em saúde pública veterinária se reuniram no final de 1974, em Genebra. O principal objetivo do encontro era reforçar a importância cada vez maior dos médicos veterinários no trabalho de saúde pública e a 
conseqüente necessidade de fortalecer os serviços de saúde pública veterinária. Na reunião, foram definidos os propósitos do campo de atuação: "A saúde pública Veterinária (SPV) é um componente das atividades de saúde pública devotado à aplicação das habilidades, conhecimentos e recursos da profissão veterinária para a proteção e melhora da saúde humana" (WORLD HEALTH ORGANIZATION, 1975).

No documento, há uma nota explicativa afirmando que a medicina veterinária é um braço estendido da medicina, que se ocupa da saúde de outras espécies animais que não os seres humanos. A saúde pública veterinária desempenha diversas funções na saúde pública que obedecem à vasta comunhão de interesses existentes entre a medicina veterinária e a medicina humana e oferecem a oportunidade de uma proveitosa interação entre ambas. Como profissão cruzada, a saúde pública veterinária apresenta natureza interdisciplinar, voltando-se simultaneamente para ambas as direções: os seres humanos e os animais.

A produção de proteínas de alto valor biológico para consumo humano em quantidade suficiente é resultado do sucesso da Medicina Veterinária em manter economicamente sob controle as doenças animais. Essas atividades são importantes benefícios adicionais na proteção da saúde humana, especialmente quando os esforços no controle de doenças se direciona para o combate às zoonoses (WORLD HEALTH ORGANIZATION, 1975).

Atualmente, as atividades básicas de proteção da saúde animal, com especial atenção para o combate às zoonoses fazem com que as concepções de saúde e doença da Medicina Veterinária Preventiva sejam as mesmas da saúde pública veterinária formando um modo único de pensar - a preocupação com a promoção da saúde na coletividade, constituindo um estilo de pensamento de Medicina Veterinária Preventiva e Saúde Pública (PFUETZENREITER, 2003).

Os médicos veterinários podem desempenhar dois tipos de função dentro da saúde pública. O primeiro tipo estabelece as atividades para as quais o veterinário tem uma qualificação única. $\mathrm{O}$ outro abrange as atividades que podem ser desempenhadas igualmente pelos veterinários, pelos médicos e pelos demais profissionais do setor. A publicação da OMS resultante de uma reunião de especialistas em saúde pública veterinária (WORLD HEALTH ORGANIZATION, 1975) procurou especificar essas contribuições da Medicina Veterinária para a Saúde Pública, como será detalhado a seguir.

São inúmeras as contribuições da Medicina Veterinária para a saúde humana. A primeira e mais básica função do sanitarista veterinário está fundamentada no contexto puramente veterinário por sua conexão com os animais inferiores e suas doenças, relacionado à saúde e bem-estar humanos. Essas atividades refletem as qualificações específicas dos médicos veterinários e normalmente são a base da formação do veterinário de saúde pública dos organismos de saúde.

O encargo relacionado diretamente com os animais inclui: a) diagnóstico, controle e vigilância em zoonoses; b) estudos comparativos da epidemiologia de enfermidades não infecciosas dos animais em relação aos seres humanos; c) intercâmbio de informações entre a pesquisa médica veterinária e a pesquisa médica humana com vistas à aplicação desta para as necessidades da saúde humana; d) estudo sobre substâncias tóxicas e venenos provenientes dos animais; e) inspeção de alimentos e vigilância sanitária; f) estudo de problemas de saúde relacionados às indústrias animais, incluindo o destino adequado de dejetos; g) supervisão da criação de animais de experimentação; h) estabelecimento de interligação e cooperação entre as organizações de saúde pública e veterinária com outras unidades relacionadas com animais; i) consulta técnica sobre assuntos de saúde humana relativos aos animais.

Outros contextos das atividades desempenhadas pelo sanitarista veterinário são o biomédico e o generalista. Ainda que o médico veterinário exerça atividades puramente veterinárias como as acima mencionadas, sua ampla formação básica nas ciências biomédicas o qualifica para desempenhar muitos papéis adicionais na saúde pública, que são comuns aos médicos e a outros membros da equipe como: a) epidemiologia em geral; b) laboratório de saúde pública; c) produção e controle de produtos biológicos; d) proteção dos alimentos; e) avaliação e controle de medicamentos; f) saneamento ambiental; g) pesquisa de saúde pública.

A formação conferida aos médicos veterinários os qualifica para desempenhar funções generalistas, que podem ser executadas por outros membros da equipe de saúde pública, como a administração, o planejamento e a coordenação de programas de saúde pública.

De acordo com o Comitê de Especialistas em Saúde Pública Veterinária (WORLD HEALTH ORGANIZATION, 1975) o ponto crucial de implementar a ampliação da atuação do médico veterinário na saúde pública está não apenas em 
melhorar os canais de comunicação interprofissionais e em estabelecer uma infra-estrutura apropriada para a carreira, mas sobretudo, em assegurar uma boa formação aos profissionais na área. Deve haver um estímulo e fortalecimento da educação veterinária que deveria receber apoio tanto por parte dos setores de saúde pública, quanto da agricultura - para o ensino de saúde pública. Todos os profissionais deveriam estar voltados para a importância da profissão veterinária para a saúde humana, sendo considerado lamentável quando um médico veterinário não está consciente disso.

\section{CENÁRIO ATUAL E TENDÊNCIAS DA MEDICINA VETERINÁRIA PREVENTIVA E SAÚDE PÚBLICA}

A expressão saúde pública veterinária é utilizada para designar o marco conceitual e a estrutura de implementação das atividades de saúde pública que empregam conhecimentos e recursos da medicina veterinária para proteger e melhorar a saúde humana. A saúde pública veterinária vincula a agricultura, a saúde animal, a educação, o ambiente e a saúde humana. Seus princípios de base estão fortemente ligados nas ciências biológicas e sociais que se encontram amplamente difundidos na agricultura, na medicina e no meio ambiente (ARÁMBULO, 1991).

A proteção dos alimentos e o controle e erradicação de zoonoses permanecem as funções de maior interesse na área. Também ganham destaque outros três enfoques: os modelos biomédicos (pesquisas em animais para estudar os problemas de saúde dos seres humanos), o desenvolvimento dos serviços de saúde pública veterinária, e o ensino e formação em saúde pública. Em relação ao último tópico, recomenda-se a mudança de abordagem dos currículos - com concentração excessiva na clínica para fornecer uma educação mais voltada para os aspectos de saúde pública (ARÁMBULO, 1991).

BÖGEL (1992) aponta para a importância da realização de pesquisas sobre as necessidades e tendências da educação veterinária e confirma a atenção que deve ser dispensada à formação veterinária enfatizando a saúde pública. O autor argumenta que em um mundo com uma população cada vez mais numerosa, que recorre a novos sistemas de exploração do solo e a novas tecnologias, é importante o desenvolvimento de uma medicina veterinária populacional. A orientação dispensada à medicina veterinária dentro da tríade formada pelo meio ambiente, o animal e o homem deve ser acompanhada de uma importante expansão da saúde pública veterinária e de uma profunda modificação da formação veterinária, mais centrada na interdisciplinaridade. O autor aponta que os principais problemas enfrentados pela saúde pública veterinária são as novas biotecnologias, o controle das infecções de origem alimentar, os novos sistemas de exploração agrária e as questões éticas relativas a esses problemas.

Tradicionalmente dentro do âmbito da saúde pública, a medicina veterinária tem trabalhado no controle das zoonoses e na proteção sanitária dos alimentos. Além destes setores, situações específicas relacionadas com o meio ambiente têm chamado a atenção para a atuação da profissão veterinária. $\mathrm{O}$ trabalho interdisciplinar, a incorporação nos grupos inter-setoriais e inter-institucionais que planificam, executam e avaliam estudos e projetos de impacto ambiental, estão abrindo oportunidades para a presença do médico veterinário nesse segmento (CIFUENTES, 1992).

Para realizar atividades ligadas à área ambiental, CIFUENTES (1992) aponta que o médico veterinário deve ter conhecimentos gerais sobre as ciências do ambiente, além de conhecimentos sobre: a) as relações ambiente-enfermidade; b) as atividades agropecuárias e suas relações sobre o ambiente; c) modelos de avaliação de estudos de impacto ambiental; d) tecnologia básica para a proteção e saneamento ambiental. Na formação acadêmica dos médicos veterinários, o autor propõe que as escolas ofereçam conhecimentos aprofundados nas áreas de ciências ambientais, ecologia, biologia e saneamento ambiental para que os profissionais possam ser incorporados e oferecer sua contribuição a esses setores.

NIELSEN (1997) declara que o profissional de Medicina Veterinária deve ter um nível de competência consistente com as demandas da sociedade. O reconhecimento da importância da profissão para a sociedade está na dependência de sua relevância social. As questões de maior relevância social apontadas para a profissão para este século são: a) produção de alimentos com utilização de métodos sustentáveis levando em consideração o crescimento populacional; b) proteção do meio ambiente à degradação e perda da biodiversidade; c) profilaxia das novas zoonoses com potencial epidêmico. Todas essas questões apontadas pelo autor estão ligadas à sustentabilidade.

A redução da pobreza especialmente nas comunidades rurais, a produção de alimentos sem produzir desgaste ambiental e o controle de 
enfermidades relacionadas ao meio ambiente constituem alguns dos desafios para a população mundial atual. Nesse contexto cresce a importância da participação do sanitarista veterinário nessas questões ligadas à sustentabilidade, em que as populações devem examinar seus padrões de produção e consumo e se comprometer com um crescimento econômico responsável que respeite o meio ambiente. Todos esses tópicos ligados à sustentabilidade devem ser mais enfatizados na formação veterinária.

O problema das zoonoses é destacado por vários autores (CRIPPS, 2000; OSBURN, 1996; STÖHR \& MESLIN, 1997). Esse grupo de enfermidades continua a representar um importante problema de saúde para grande parte do mundo, com elevadas perdas para os setores de saúde e de agricultura, principalmente nos países em desenvolvimento. O risco de infecções emergentes por novas entidades patológicas ou por agentes conhecidos aparecendo em novas áreas ou em novas condições vem aumentando nos últimos anos. O controle das enfermidades desta natureza requer uma cooperação inter-setorial e inter-institucional, reunindo segmentos ligados à saúde, finanças, planejamento, comércio, agricultura e indústria de alimentos, consumidores e comunidade científica biomédica e agrária (STÖHR \& MESLIN, 1997).

Em 1999, reuniu-se na Itália um Grupo de Estudos para discutir as tendências da Saúde Pública Veterinária para o futuro. Na publicação resultante do encontro (WORLD HEALTH ORGANIZATION, 2002) está contida uma revisão que se tornou uma oportunidade de reexame do papel e funções sobre a área.

A preocupação predominante da Saúde Pública Veterinária durante os anos de 1970 e 1980 estava voltada para os riscos da poluição química ao ambiente e aos alimentos (resultante de pesticidas, resíduos animais, e outras substâncias tóxicas). Entretanto, nas duas últimas décadas as zoonoses emergentes e re-emergentes têm adquirido significância global. Dentre outros, são citados os problemas relacionados à Salmonella enteritidis das aves, as febres hemorrágicas virais de Marburg e Ebola, a ligação entre a encefalite espongiforme bovina e a doença de Creutzfeldt-Jacob, as Hantaviroses, e vários outros exemplos de agentes zoonóticos que requerem o trabalho conjunto de médicos, veterinários e biólogos. Ao lado desses problemas estão as novas tendências na prática de produção, as interferências nas populações de animais silvestres, as mudanças demográficas, a mobilidade das populações, a urbanização e globalização da indústria de alimentos. Essas alterações devem estar acompanhadas de níveis aumentados de vigilância epidemiológica e de novas abordagens para o controle e prevenção de doenças (WORLD HEALTH ORGANIZATION, 2002).

Em face dessa nova situação, as atividades da Saúde Pública Veterinária devem estar em consonância com outros esforços nas áreas da saúde, agricultura e ambiente. O Grupo de Estudos redefiniu a Saúde Pública Veterinária e o escopo de seus esforços colaborativos, e a Saúde Pública Veterinária passou a ser considerada como "a soma de todas as contribuições para o bem-estar físico, mental e social dos seres humanos mediante a compreensão e aplicação da ciência veterinária" (WORLD HEALTH ORGANIZATION, 2002). A ciência veterinária engloba todas as atividades veterinárias incluindo a produção animal e a saúde, cumprindo as funções essenciais na saúde pública e influenciando diretamente a saúde humana pelo seu conhecimento e experiência.

Pelo menos metade dos 1700 agentes conhecidos que infectam os seres humanos tem um vertebrado como reservatório animal ou inseto como vetor e muitas doenças emergentes são zoonoses. Em vista disso, há necessidade de fortalecimento da ligação entre a medicina animal e a humana. Além das atividades habituais, os domínios específicos emergentes da Saúde Pública Veterinária que podem trazer contribuições significativas para a saúde pública são: a) investigação, epidemiologia e controle de doenças comunicáveis não zoonóticas; b) aspectos sociais, comportamentais e mentais da relação entre seres humanos e animais; c) epidemiologia e prevenção de doenças não infecciosas (incluindo a promoção de estilos de vida saudáveis); d) análises e avaliações de serviços e programas de saúde pública; e) atividades que envolvem o contexto social, especialmente aquelas em que há participação em programas de educação em saúde (WORLD HEALTH ORGANIZATION, 2002).

Deve ser ressaltada uma atividade importante do médico veterinário dentro da saúde pública que é a educação em saúde. Esse profissional pode atuar na difusão de informações e na conscientização das pessoas sobre os temas ligados à saúde. A participação do sanitarista veterinário é fundamental nos programas de educação em saúde para a proteção e promoção da saúde humana em comunidades dentro dos princípios do desenvolvimento sustentável.

Esse significativo tema, ao lado daqueles discutidos aqui anteriormente, deve ser trabalhado com atenção na formação veterinária para 
acompanhar as necessidades atuais da sociedade e antecipar as exigências para o futuro. $\mathrm{O}$ profissional formado em Medicina Veterinária que possuir sólidos fundamentos nos conteúdos pertinentes à Medicina Veterinária Preventiva e Saúde Pública, além da habilidade para trabalhar de forma interdisciplinar estará preparado para auxiliar as populações humanas a enfrentarem seus principais desafios.

\section{REFERÊNCIAS BIBLIOGRÁFICAS}

ARÁMBULO, P.V. Veterinary public health: perspectives at the threshold of the $21^{\text {st }}$ century. Revue Scientific Technique, v.11, n.1, p.255-262, 1991.

BÖGEL, K. Veterinary public health perspectives: trend assessment and recommendations. Revue Scientific Technique, v.11, n.1, p.219-239, 1992.

CIFUENTES, E.E. Protección del medio ambiente y actividades de salud pública veterinaria. Revue Scientifique Technique, v.11, n.1, p.191-203, 1992.

CRIPS, P.J. Veterinary education, zoonoses and public health: a personal perspective. Acta Tropica, v.76, p.77-80, 2000.

NIELSEN, N.O. Reshaping the veterinary medical profession for the next century. Journal of American Veterinary Medical Association, v.210, n.9, p.1272-1274, 1997.

ORGANIZACIÓN MUNDIAL DE LA SALUD. Grupo consultivo sobre veterinaria de salud publica. Geneva : WHO, 1957. 30p. (Informe Técnico n.111).

ORGANIZACIÓN PANAMERICANA DE LA SALUD. A competency-based curriculum for veterinary public health and preventive medicine. Washington: Paho/WHO, 1975. 115p. (Publicación Científica 313).

OSBURN, B.I. Emerging diseases with a worldwide impact and the consequences for veterinary curricula. Veterinary Quarterly, v.18, n.3, p.124-126, 1996.

PFUETZENREITER, M.R. O ensino da medicina veterinária preventiva e saúde pública nos cursos de medicina veterinária - estudo de caso realizado na Universidade do Estado de Santa Catarina. Florianópolis, 2003. 459f. Tese (Doutorado em Educação) - Programa de Pós-graduação em Educação, Universidade Federal de Santa Catarina.

ROSEN, G. Uma história da saúde pública. São Paulo : Hucitec, 1994. 413p.

SCHWABE, C.W. Veterinary medicine and human health. 3.ed. Baltimore : Williams \& Wilkins, 1984. 680p.

SCHWABE, C.W. History of the scientific relationships of veterinary public health. Rev Sci Tech, v.10, n.4, p.933-949, 1991.

STÖHR, K.; MESLIN, F.X. The role of veterinary public health in the prevention of zoonoses, Arch Virol, v.13, suppl.1, p.207-218, 1997.

VIANNA PAIM, G.; CAVALCANTE DE QUEIROZ, J. Uma definição para saúde pública veterinária. Boletín de la Oficina Sanitaria Panamericana, v.69, n.2, p.166-168, 1970.

WORLD HEALTH ORGANIZATION. Joint WHO/FAO Expert Group on Zoonoses - Report on the First Session, Geneva, 1951. 47p. (Technical Report Series n.40).

WORLD HEALTH ORGANIZATION. Joint WHO/FAO Expert Committee on Zoonoses - Second Report. Geneva, 1959. 83p. (Technical Report Series n. 169).

WORLD HEALTH ORGANIZATION. The veterinary contribution to public health practice. Report of a Joint FAO/ WHO Expert Committee on Veterinary Public Health. Geneva, 1975. 79p. (Technical Report Series n.573).

WORLD HELTH ORGANIZATION. Future Trends in Veterinary Public Health. Report of a WHO Study Group. Geneva, 2002. 85p. (WHO Technical Report Series n.907). 